\title{
Plasma disappearance of glycated and non-glycated albumin in Type 1 (insulin-dependent) diabetes mellitus: evidence for charge dependent alterations of the plasma to lymph pathway
}

\author{
L. Bent-Hansen, B. Feldt-Rasmussen, A. Kverneland and T. Deckert \\ Steno Diabetes Center, Gentofte, Denmark
}

\begin{abstract}
Summary. The fractional plasma escape rates of glycated and non-glycated albumin have earlier been measured in groups of Type 1 (insulin-dependent) diabetic patients and control subjects. The escape of non-glycated albumin was similar in control subjects and normoalbuminuric patients, but elevated in patients with micro or macroalbuminuria. In all groups the escape rate of glycated albumin was lower than that of non-glycated albumin. Glycation increases the anionic charge of albumin. To assay for charge-dependent alterations of transport a selectivity index (non-glycated albumin/glycated albumin transport ratio) was determined from the disappearance data. The index was high in control subjects $(1.021 \pm 0.0057$ (SEM)). This reflects a mean difference between the two escape rates of $2.1 \%$ per hour (for comparison the mean of the fractional escape rate of non-glycated albumin of the normal control subjects was $4.7 \%$ per
\end{abstract}

hour). The index was numerically even higher in normoalbuminuric patients $(1.031 \pm 0.0047$ (SEM)), but reached significantly lower levels in patients with microalbuminuria $(1.013 \pm 0.0030$ (SEM), $p<0.02)$. Patients with clinical nephropathy had very low levels indicating loss of selectivity $(1.002 \pm 0.0068$ (SEM), $p<0.001)$. This pattern accords well with measurements of renal clearance selectivity indices, suggesting a general, progressive deterioration of anionic perivascular barrier components in diabetic microangiopathy. The structural target for these changes is likely to be the glycosaminoglycans of the glomerular basal membrane and the interstitial matrix.

Key words: Albumin, capillary permeability, glycated albumin, diabetes mellitus.
Plasma protein disappearance is elevated in Type 1 (insulin-dependent) diabetic patients with microalbuminuria, reflecting a general deterioration of the microvascular barrier function when entering this stage of disease $[1,2]$. However, the specificity of the lesion and the relation to structural or hydrodynamic alterations is unknown.

In 1987 we studied the plasma disappearance of glycated and non-glycated albumin in Type 1 diabetic patients with normal or elevated urinary albumin excretion rates [3]. The primary aim was to investigate potential metabolic differences, but crude transport data were included as well. We observed a selective restriction of the plasma disappearance of the more electronegative glycated albumin compared with non-glycated albumin in healthy control subjects compared with Type 1 diabetic patients. There were no significant differences between the three diabetic subgroups which were established on the basis of their urinary albumin excretion rates. Based on the recent demonstrations of increased charge dependent glomerular transport in the presence of microal- buminuria [4-6] we recalculated our previous data in order to assay the charge specificity of increased whole body plasma protein disappearance in Type 1 diabetic patients with or without signs of increased glomerular protein sieving.

\section{Subjects and methods}

The experiment has been described before [3]. Twenty-five male Type 1 diabetic patients were divided into groups according to their urinary albumin excretion in three or more 24 -h urine collections. Group 1 had normal albumin excretion ( $<30 \mathrm{mg} / 24 \mathrm{~h}$ ), group $2 \mathrm{had}$ microalbuminuria $(30-300 \mathrm{mg} / 24 \mathrm{~h})$ and group $3 \mathrm{had}$ clinical nephropathy ( $>300 \mathrm{mg} / 24 \mathrm{~h}$ ). Six normal male subjects, matched for age with the diabetic groups served as the control group (age mean: $32 \pm 8$ years).

At time zero $80 \mathrm{kBq}$ of ${ }^{131} \mathrm{I}$-albumin and $80 \mathrm{kBq}$ of ${ }^{125} \mathrm{I}$-glycated albumin ( $5.5 \mathrm{~mol}$ glucose $/ \mathrm{mol}$ albumin) was injected in one arm. Plasma samples were taken every $10 \mathrm{~min}$ for the first hour following injection.

The plasma samples were assayed for the content of ${ }^{131} \mathrm{I}$ and ${ }^{125} \mathrm{I}$ activity in a dual channel scintillation counter. 
Table 1. Results

\begin{tabular}{lll}
\hline & $\begin{array}{l}\mathrm{FER}_{\text {non-glycated albumin }} \\
\left(\% \cdot \mathrm{h}^{-1}\right)\end{array}$ & $\begin{array}{l}\text { Selectivity index } \\
\left(\mathrm{h}^{-1}\right)\end{array}$ \\
\hline $\begin{array}{l}\text { Control subjects } \\
(n=6)\end{array}$ & $5.74 \pm 0.68$ & $1.021 \pm 0.0057$ \\
$\begin{array}{l}\text { Normoalbuminuria } \\
(n=9)\end{array}$ & $5.60 \pm 0.67$ & $1.031 \pm 0.0050$ \\
$\begin{array}{l}\text { Microalbuminuria } \\
(n=8)\end{array}$ & $7.57 \pm 0.72^{\mathrm{a}, \mathrm{b}}$ & $1.014 \pm 0.0030^{\mathrm{b}}$ \\
$\begin{array}{l}\text { Clinical nephropathy } \\
(n=8)\end{array}$ & $9.14 \pm 0.88^{\mathrm{a}, \mathrm{b}}$ & $1.004 \pm 0.0068^{\mathrm{a}}, \mathrm{b}$ \\
\hline
\end{tabular}

${ }^{a} p<0.05$ vs control subjects

${ }^{\circ} p<0.05$ vs normoalbuminuric patients

$\mathrm{FER}_{\mathrm{a}}$, Fractional escape rate of non-glycated albumin

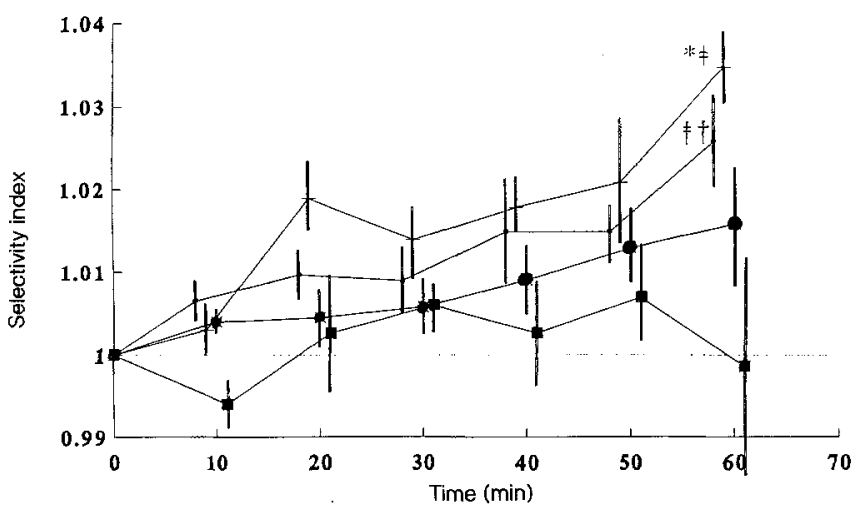

Fig. 1. The ratio of plasma non-glycated albumin/glycated albumin in the three patient groups and in control subjects (mean \pm SEM). - normal control subjects, patients with: + normal urinary albumin excretion rate, - microalbuminuria, diabetic nephropathy. $* p<0.001+p<0.05$ vs patients with microalbuminuria; $\neq p<0.001$ vs patients with nephropathy

\section{Calculations}

In order to compensate for small changes in plasma water during the experiment the plasma activity was divided with the total protein of the sample. The disappearance curves were drawn in a semilogarithmic scale, and the fractional escape rate $\left(\mathrm{FER}_{\text {abumin }}\right)$ was determined as the time constant of the ${ }^{131} I$ curves from 10 to $60 \mathrm{~min}$. The ${ }^{131} \mathrm{I} /{ }^{125} \mathrm{I}$ ratio was calculated at each point, and correlated with time (method of least squares). From these correlation lines the selectivity index (SI) was calculated by dividing the zero ratio of each line with the 60-min value. Thus the SI of each subject was determined from all six measured plasma ratios, denoting the increase of $F R_{\text {non-giycated }}$ alburtin over FER glycated ajbumin. The direct comparison of the simultaneously determined plasma activities instead of the calculated FERnon-glycated albumin and FER glycated albumin [3] yields a much more sensitive analysis, cancelling some of the considerable variation in the calculation of FER.

\section{Statistical analysis}

Results are given as mean \pm SEM. The Student's $t$-test was used for comparison between the groups. A $p$ value of $<0.05$ (two-tailed) was considered significant.

\section{Results}

Results are shown in Table 1 and Figure 1 . The FER non-gly- $_{\text {- }}$ cated albumin as originally calculated is given in the first column, and the calculated SI in the second. The figure 1.021 denotes an absolute difference between the two FER values (\% per hour) of $2.1 \%$ per hour. It appears that only in group 3 does the SI approach 1, indicating similar transport of the two albumins. In all other groups the transport of albumin was faster than that of glycated albumin (SI $>1, p<0.005$ ).

It should be noted, that the calculation of FER $R_{\text {non-glycated }}$ albumin and SI only describes the plasma disappearance curves from 10 to $60 \mathrm{~min}$. To see if there were any charge-dependent effects in the initial part of the curve from zero to $10 \mathrm{~min}$, the zero ratio of the correlation lines was divided by the standard ratio of the injectate. However, no significant changes were found.

\section{Discussion}

Glycation of albumin takes place at the free lysine amino terminals, thereby increasing the net anionic charge of the molecule [7]. The size increases to a lesser degree, making the simultaneous transport index i.e. the SI of the two albumins a sensitive tool for charge-dependent permeability analysis. In the kidney, the glomerular basement membrane functions not only as a simple molecular sieve, but also as an electrostatic barrier $[4-6,8,9]$. When applying SI clearance measurements in groups of diabetic patients and control subjects a complicated pattern appears [5]. Patients with normal urinary albumin excretion have a higher SI (non-glycated albumin clearance/glycated albumin clearance) than control subjects, patients with microalbuminuria have an SI comparable to control subjects, and patients with clinical nephropathy have a low SI. In concert with other relative clearance data $[6,9]$ these findings indicate a basic decrease of charge-dependent transport in normoalbuminuric diabetic patients. This may be caused by increased glycation of membrane structures, increasing the charge density of the filter in normoalbuminuric patients. In microalbuminuria the normal electrostatic barrier function starts to degenerate, and is followed by more extensive structural lesions in overt nephropathy. The electrostatic properties of the glomerular membrane are partly due to a high content of strongly anionic glycosaminoglycans (GAGs), and an impaired synthesis of these compounds may initiate the development of diabetic nephropathy [8].

In the present study a similar pattern could be demonstrated in whole body plasma disappearance SI, suggesting that similar mechanisms operate not only in the kidney, but also in the entire peripheral circulation.

To calculate the SI we normally use the FER of the more electronegative molecule as the denomonator i. e. $\mathrm{SI}=\mathrm{FER}_{\text {non-glycated albumin }} / \mathrm{FER}_{\text {glycated albumin }}$. By this calculation the: $(S I-1) \times 100$ equals the relative difference between the FER values; the unit is: "percent of the FER $\mathrm{gly}_{\text {- }}$ cated albumin". (In the original paper [3] the SI was given as the reciprocal value). The equation in that paper was based on the individually estimated FER values which resulted in a high risk for type 2 error due to the high variability of each of the two estimates in combination with the small numbers studied. When using our original calculations we found that normal control subjects had a significantly 
higher index (a more electronegative barrier) than diabetic patients. The SI of normal control subjects was 1.25 indicating that the FER of the glycated albumin was increased by $25 \%$ compared with the non-glycated albumin. The corresponding value in the patients was 1.07 $(p<0.05)$. There was no significant difference between the patient groups. In the present paper we calculated for each time point the ratio between the two albumins. The SI for each patient was given as the 60 -min value estimated on the basis of the SI-correlation line of the patient. By this calculation the $(\mathrm{SI}-1) \times 100$ equals the difference in absolute terms between the FER values; the unit is: "\% per hour". The differences between the SI values in the patient groups are significant only after $60 \mathrm{~min}$ of study. However, the general trend in each of the curves seems to support the conclusion obtained on the basis of the correlation line estimates.

As recently shown [10], the plasma disappearance of albumin includes two phases: one fast in the interval from zero to about $10 \mathrm{~min}$, and one slow in the interval from 10 to $60 \mathrm{~min}$ (FER). The first phase is believed to mainly denote the tracer passage of capillary walls, and the latter the interstitial transport. However, the increased permeability in diabetic patients with albuminuria $[2,6,9]$ as well as the changes of SI seem limited to the second phase, and may thus reflect interstitial rather than endothelial alterations. Similar to the glomerular basement membrane, a major resistance component of extracellular space to macromolecules is the content of anionic GAGs. These are chemically closely related to the renal GAGs, and although they do not have a firm structure and differ in composition and charge, they probably share the same metabolic pathways. Thus, a generalized default synthesis of highly charged GAGs in susceptible patients is a likely basic step in the formation of malignant diabetic microangiopathy. Apart from the barrier function, GAGs have strong anticoagulative and anti-precipitating capabilities, and participate in microvascular as well as macrovascular repair processes. A defect synthesis of GAGs might therefore explain aspects of increased hyalinization, fibrosis, and atherosclerosis in albuminuric patients.

In conclusion, the peripheral SI of albumin/glycated albumin disappearance produces a similar pattern as in the kidney. This indicates, that the peripheral barrier to macromolecules is also a charged barrier, and that an early step in the development of diabetic microangiopathy is the break-down of this barrier.

\section{References}

1. Parving H-H, Rasmussen SM (1973) Transcapillary escape rate of albumin in short- and long-term juvenile diabetics. Scand J Clin Lab Invest 32: $81-87$

2. Feldt-Rasmussen B (1986) Increased transcapillary escape rate of albumin in type 1 (insulin-dependent) diabetic patients with microalbuminuria. Diabetologia 29: 282-286

3. Bent-Hansen L, Feldt-Rasmussen B, Kverneland A, Deckert T (1987) Transcapillary escape rate and relative metabolic clearance of glycated and non-glycated albumin in type 1 (insulin-dependent) diabetes mellitus. Diabetologia 30: $2-4$

4. Viberti GC, Mackintosh D, Keen H (1983) Determinants of the penetration of proteins through the glomerular barrier in insulindependent diabetes mellitus. Diabetes 32 [Suppl 2]: 92-95

5. Kverneland A, Feldt-Rasmussen B, Vidal P et al. (1986) Evidence of changes in renal charge selectivity in patients with type 1 (insulin-dependent) diabetes mellitus. Diabetologia 29: 634-639

6. Deckert T, Kofoed-Enevoldsen A, Vidal P, Nørgaard K, Andreassen HB, Feldt-Rasmussen B (1993) Size and charge selectivity of glomerular filtration in type 1 (insulin-dependent) diabetic patients with and without albuminuria. Diabetologia 36 : 244-251

7. Vidal P, Deckert T, Hansen B, Welinder WS (1989) High performance liquid chromatofocusing and column affinity chromatography of in vitro ${ }^{14} \mathrm{C}$-glycated human serum albumin. Demonstration of a glycation-induced anionic heterogeneity. J Chromatogr 476: $467-475$

8. Rennke HFG, Cotran RS, Venkatachalam MA (1975) Role of molecular charge in glomerular permeability: tracer studies with cationized ferritins. J Cell Biol 67: 638-643

9. Deckert T, Feldt-Rasmussen B, Djurup R, Deckert M (1988) Glomerular size and charge selectivity in insulin-dependent diabetes mellitus. Kidney Int 33: 100-106

10. Bent-Hansen L (1991) Initial plasma disappearance and tissue uptake of ${ }^{131} \mathrm{I}$-albumin in normal rabbits. Microvasc Res 41:345356

Received: 25 November 1992

Dr. L. Bent-Hansen

Cardiovascular Laboratory (2014)

Medical Department B

Rigshospitalet

DK-2100 Copenhagen $\varnothing$

Denmark 